\title{
A Framework and Taxonomy of Videogame Playing Preferences
}

\author{
Gustavo F. Tondello ${ }^{1,2}$, Rina R. Wehbe ${ }^{1,2}$, Rita Orji ${ }^{1,4}$, Giovanni Ribeiro ${ }^{1}$, Lennart E. Nacke ${ }^{1,2,3}$ \\ ${ }^{1} \mathrm{HCI}$ Games Group, Games Institute, ${ }^{2}$ Cheriton School of Computer Science, ${ }^{3}$ Drama and Speech \\ Communication, Stratford Campus, University of Waterloo \\ ${ }^{4}$ Faculty of Computer Science, Dalhousie University \\ gustavo@tondello.com,rina.wehbe@uwaterloo.ca,rita.orji@dal.ca,ggsribei@uwaterloo.ca, lennart.nacke@acm.org
}

\begin{abstract}
Player preferences for different gaming styles or game elements has been a topic of interest in human-computer interaction for over a decade. However, current models suggested by the extant literature are generally based on classifying abstract gaming motivations or player archetypes. These concepts do not directly map onto the building blocks of games, taking away from the utility of the findings. To address this issue, we propose a conceptual framework of player preferences based on two dimensions: game elements and game playing styles. To investigate these two concepts, we conducted an exploratory empirical investigation of player preferences, which allowed us to create a taxonomy of nine groups of game elements and five groups of game playing styles. These two concepts are foundational to games, which means that our model can be used by designers to create games that are tailored to their target audience. In addition, we demonstrate that there are significant effects of gender and age on participants' preferences and discuss the implications of these findings.
\end{abstract}

\section{ACM Classification Keywords}

H.1.2. User/Machine Systems: Human Factors; H.5.m. Information Interfaces and Presentation (e.g. HCI): Miscellaneous; K.8.0. Personal Computing: Games

\section{Author Keywords}

Games User Research; Game Elements; Player Types.

\section{INTRODUCTION}

Understanding how players differ in their preferences for various game elements and playing styles has many practical uses. For example, game designers can use this information to better target their ideas towards specific groups of players or towards broader audiences, whereas marketers can better understand their target players and direct their communication efforts accordingly.

\footnotetext{
Permission to make digital or hard copies of all or part of this work for personal or classroom use is granted without fee provided that copies are not made or distributed for profit or commercial advantage and that copies bear this notice and the full citation on the first page. Copyrights for components of this work owned by others than the author(s) must be honored. Abstracting with credit is permitted. To copy otherwise, or republish, to post on servers or to redistribute to lists, requires prior specific permission and/or a fee. Request permissions from permissions@ acm.org.

CHI PLAY '17, October 15-18, 2017, Amsterdam, Netherlands

(C) 2017 Copyright held by the owner/author(s). Publication rights licensed to ACM ISBN 978-1-4503-4898-0/17/10 . \$ \$15.00
}

DOI: https : //doi .org/10.1145/3116595. 3116629
For this purpose, several researchers have suggested models of player types or gaming motivations. These models are generally based on conceptual analysis, psychographic methods, or behavioural analysis [17]. However, researchers Hamari and Tuunanen [17] have noted that most of these approaches suffer from similar limitations. First, most of them were based on or were inspired by Bartle's original work [2], which suffers a number of limitations. Bartle conducted a qualitative and conceptual study of multi-user dungeons (MUD) players to classify them in types. However, his work was never empirically validated nor intended to be used outside of the context of MUDs. Nevertheless, it inspired and became the foundation of most of the posterior attempts to create player typologies for multiple genres. Consequently, many of the proposed models are limited in the number and types of motivational factors considered because most constructs are similar or derivatives of Bartle's four types. Another issue is that most studies were limited to online games or massively multiplayer online games (MMOs). This makes it difficult to transfer insights to other game genres or contexts. Finally, many models attempt to classify players in distinct types [3,24]. This can be misleading because they try to segment players in dichotomous categories. This approach might not adequately represent reality because humans are complex and players usually display a combination of distinct preferences in variate degrees, instead of a single category of preferences.

To address these issues, we present a novel conceptual framework and an associated taxonomy of videogame play preferences. Rather than using Bartle's taxonomy or one of its derived works as a building block, we devised a new framework based on existing game design literature. We considered player preferences from the point of view of two distinct constructs in our conceptual framework: game elements and game playing styles. Game elements are the activities that players engage with in games, such as resource management, exploration, or combat. Game playing styles, on the other hand, refer to the different ways by which players interact with the game and with other players, instead of the activities that players do. Hence, we studied game playing styles such as team-based play, competitive play, solo play, and e-sports. Next, we created a new taxonomy by analyzing players' preferences from empirical data related to a broad range of game 
elements and playing styles. This way, we explored diverse elements and styles that were never previously investigated.

Our work contributes to the human-computer interaction (HCI) and games user research (GUR) communities by developing a new conceptual framework and taxonomy for understanding player preferences and hence, addressing the shortcomings of prior works. Moreover, we demonstrate that participants' gender and age significantly influence their preferred elements and play styles. Our framework is applicable for designing and studying a broader category of games, game elements, and playing styles and is grounded on solid empirical evidence. Therefore, it can be used to inform the design of more engaging games because it helps designers understand which elements and playing styles players usually enjoy when playing together. Furthermore, detailed information about the target audience of a game is necessary because of an increasing interest in the design of serious games (i.e., games that serve a main purpose other than entertainment, such as health, education or fostering awareness to a cause). Particularly, for serious games it is important to understand how players are motivated by different gaming elements and styles, so the game can achieve its instrumental purpose for distinct players.

The remainder of this work is organized as follows. First, we review the existing literature on player typologies and preferences. Next, we introduce our player preferences framework and the results of an empirical study in which we analyzed participants' self-reported preferences towards 66 game elements and 20 game playing styles to devise a taxonomy or player preferences. Finally, we discuss the implications of our findings and the potential applications of the framework.

\section{RELATED WORK}

\section{Player Typologies and Preferences}

In the last decade, researchers in games and HCI have been studying different game play motivations and playing styles and representing them in player typologies. Bartle's original player types for MUDs [2] are based on two axes that express the player's desire to interact with or act on the virtual world or other players: ACHIEVERS (acting on the world), EXPLORERS (interacting with the world), SOCIALISERS (interacting with other players), and KILLERS (acting on other players). Bartle later extended it by adding a third dimension [3]: implicit/explicit (i.e., whether the player actions are automatic and unconscious or considered and planned). Thus, each of the four original types was split in two subtypes. The implicit subtypes are respectively OPPORTUNISTS, HACKERS, FRIENDS, and GRIEFERS. The explicit sub-types are respectively PLANNERS, SCIENTISTS, NETWORKERS, and POLITICIANS.

Bartle's research is a cornerstone of player typology with many researchers building off the ideas; however many researchers also expanded on it by proposing their own models. Based on a factor analysis of questions inspired by Bartle's player types, Yee $[35,38]$ identified three main components of player motivation with ten subcomponents: ACHIEVEMENT (advancement, mechanics, competition), SOCIAL (socializing, relationship, teamwork), and IMMERSION (discovery, roleplaying, customization, escapism). His analysis provides a solid base for understanding player motivation, but it is limited because of its focus on one specific game genre (Massively Multiplayer Online Role-Playing Games-MMORPGs).

Bateman and Boon [4] contributed research to provide a wider perspective regarding player types by adapting the MyersBriggs Type Indicator (MBTI, [23]) to games. Their first demographic game design model (DGD1) proposed the player styles CONQUEROR, MANAGER, WANDERER, and PARTICIPANT. The second demographic game design model (DGD2, [5]) explored the hardcore to casual dimension, different skill sets, and the preference for single and multiplayer gameplay. Following this work, the BrainHex [5, 24] was developed, which is a top-down player typology that takes inspiration from neurobiological player satisfaction research [6], previous player typologies, discussions of patterns of play, and the literature on game emotions to build seven archetypes denoting distinct experiences of play. The BrainHex archetypes are: ACHIEVERS (goal-oriented and motivated by completion); CONQUERORS (enjoy struggling against strong opponents); DAREDEVILS (motivated by excitement and risk taking); MASTERMINDS (enjoy solving puzzles and devising strategies); SEEKERS (motivated to explore the game world); SOCIALISERS (enjoy interacting with other people); and SURVIVORS (enjoy frightening experiences in games).

Busch et al. [11] conducted a validation of the BrainHex model and argued for an improvement of its psychometric properties, based on poor results of factor, stability, and internal consistency analyses. Despite lack of empirical validation, the BrainHex is increasingly gaining researchers' attention; it has been used by several studies for investigating player motivation and personalization of games. For example, Orji et al. [26] used BrainHex to tailor persuasive health games to different player types and Birk et al. [8] used BrainHex to model player motivation in a social network game.

Hamari and Tuunanen [17] conducted a systematic review of player type models to investigate their commonalities. The authors note that MMOs and online games are more frequently covered than other genres in several of these studies, therefore compromising the applicability of these models outside of the multiplayer domain. Furthermore, they compared all the analyzed models and suggested that they could be synthesized in five key dimensions of play motivations: achievement, exploration, sociability, domination, and immersion.

More recently, Yee [36] expanded on his previous work by conducting a factor analysis with over 140,000 participants and developed a gamer motivation profile composed of 12 dimensions grouped into six clusters: ACTION (destruction and excitement); SOCIAL (competition and community); MASTERY (challenge and strategy); ACHIEVEMENT (competition and power); IMMERSION (fantasy and story); and CREATIVITY (design and discovery). Yee's recent proprietary investigation intends to capture player motivations towards a large variety of games and is empirically supported by factor analysis. It is also noteworthy that it intends to classify different elements that foster different playing motivations, instead of trying to classify players in types. Unfortunately, a standard assessment tool is not publicly and readily available. 
While all these prior player preference models provide useful insights towards understanding different player motivations, most of them are limited by the lack of empirical validation, unavailability of a standard assessment tool, or are only suitable for a specific game or genre. In addition, they fail to consider different elements of play that have surfaced more recently, such as body movement-controlled games, and different styles of play, such as electronic sports, streaming, or casual games. The present work aims to fill this gap, by researching player preferences using a wider scope and providing new information based on the analyzed data.

\section{Player Preferences Based on Game Features}

Several authors have investigated how players' perceived values and identification with game characters, avatars, and objects increase motivation and enjoyment in digital games. For example, Birk et al. [7] revealed that avatar customization stimulates player identification, leading to increased autonomy, immersion, invested effort, enjoyment, and positive affect. Livingston et al. [21] interviewed twenty World of Warcraft [9] players with the goal of investigating what kind of value their game characters provided. Tondello et al. [32] and Toups et al. [33] built on their work to examine why players value and collect a diversity of game objects, including but not limited to characters. While these frameworks can help explain part of a player's preferences for different games, they are limited to one of the many aspects that define a game (their characters or objects). In contrast, our work aims to study a more generic and broad player preferences framework.

\section{Player Preferences Based on Personality Factors}

Besides models of player motivation, there is growing evidence within the HCI and GUR communities that personality factors also influence player motivation and preferences. Yee [37] investigated the relation of the five-factor personality traits [16] with the different motivations in the Gamer Motivation Profile, thus establishing a bridge between personality factors and player preferences. Similarly, Jeng and Teng [19] found a relation between personality traits and preferences for different playing styles. Likewise, Peever et al. [28] found significant correlations between personality traits and players' preferences for particular game genres. Moreover, McMahon et al. [22] investigated the relation of the five-factor personality traits with the player types of the DGD1 model. Finally, Zeigler-Hill and Monica [39] identified several significant associations between the BrainHex archetypes and the HEXACO personality traits [20]. Moreover, Orji et al. [27] studied the relation between the five-factor personality traits and several persuasive strategies used in the context of serious and persuasive games. They noted that extraversion, agreeableness, and openness are the three personality traits that predict most of the variance in the effectiveness of different persuasive strategies.

With a different approach, Ryan et al. [30] and Przybylski et al. [29] built a motivational model of video game engagement based on self-determination theory (SDT) [12]. They discovered that competence, autonomy, relatedness, presence, and intuitive controls are good predictors of player enjoyment and engagement. Their player motivation model can explain why a game is successful; however, it does not account for different player preferences.

These studies suggest that personality traits might only partly explain player preferences for different games. For example, if two games are successfully designed to equally satisfy players' psychological needs or appeal to the same personality traits, it is common for a player to still prefer one game over the other and it is not clear (from available research) what attributes make some players prefer one game over the other. Therefore, a new conceptual framework, which specifically describes player's preferences, might be more suited to explain the whole range of player motivations and play styles.

Concurrently, Ferro et al. [13] conducted a literature review and proposed a theoretical model that encompasses and suggests relationships between personality traits, player types, and game elements and mechanics. Their work identifies five combinations of these constructs: DOMINANT (which refers to players who are self-serving and want to make themselves visible), OBJECTIVIST (focus on achievement), HUMANIST (focus on social engagement), INQUISITIVE (focus on exploration and discovery), and CREATIVE (focus on creativity). The novelty of their work consists in the attempt to combine personality traits, player types, and game elements in a general model. However, their suggested model is only theoretical and still lacks empirical validation.

\section{A FRAMEWORK OF PLAYER PREFERENCES}

One of the goals of studying player types or preferences is to inform game design decisions. However, none of the approaches described in the related literature has attempted to identify the relationship between the motivational factors or player types and the elements or atoms used in the construction of games. For example, once one considers the motivation for achievement, it is not clear exactly what are the game mechanics or dynamics that afford this kind of experience. Therefore, we propose a new conceptual framework of player preferences, which is based on the elements used in the design and construction of games. Consequently, our framework will be directly applicable to informing game design.

The issue with existing attempts to describe player preferences is that they consider only high level factors, such as achievement, socialization, or immersion. Nevertheless, game designers must work on more detailed and finer levels of abstraction. They often need specific details about the building blocks that are actionable in game design. Therefore, designers and researchers have used several terminologies to describe the building blocks of games. For example, Brathwaite and Schreiber [10] refer to game atoms as the parts that make a game. These atoms include: the game state and views, the players and the elements that represent them (e.g., avatars or tokens), the mechanics (relating to the rules of the game), the dynamics (relating to the emerging patterns of play once mechanics are set in motion by players), the goals, and the theme. Schell [31], on the other hand, refers to the parts of a game as game elements. The author classifies elements into four types: the mechanics are the procedures and rules of the game, the story is the sequence of events that unfolds in the game, the aesthetics is how the game looks and feels, and the technology 
is the set of materials and interactions that make the game possible (which might be digital or not). Another approach to game design is the MECHANICS, DYNAMICS, and AESTHETICS (MDA) framework [18]. It introduces three distinct game components: MECHANICS are the smallest components of a game, DYNAMICS are the run-time behaviour of the mechanics interacting with the player, and AESTHETICS are the emotional responses or experiences evoked in the player.

Considering these diverse views on game design, we must establish what is the adequate granularity level for a conceptual framework of player preferences. We have already mentioned that we do not consider looking only at high level constructs an adequate approach because it would not explain which are the smaller building blocks that designers should use to foster different types of experiences. However, looking at the game mechanics (which represent the smallest granularity level) is also not helpful because mechanics are just generic building blocks, which are employed to build many types of dynamics and are often subject to several operationalizations. For example, a game mechanic like a token on a board might be used to build several different dynamics, such as a racing game, a strategy game, or a game of chess. Therefore, we contend that a player preferences framework must map constructs on an intermediate granularity level, which many game design methods refer as dynamics. This approach will allow us to classify player preferences in a level that can be used to inform game design by translating them to dynamics such as resource management, combat, puzzles, or racing. Therefore, we devised our conceptual framework of player preferences based on the classification of the intermediate granularity elements employed in the building of games. Nonetheless, there are many approaches to game design, which sometimes employ different terminologies, and our framework is not based on any of them in particular. Hence, we decided to employ the more generic terminology game elements (instead of dynamics) to refer to the mid-level building blocks of games that we classify in our research.

In addition to the elements that make part of a game, there are also different modes or styles or play. One example is the preference for single or multiplayer gameplay. These game playing styles are orthogonal to the game elements and can be combined with them in a variety of ways. For example, if we consider a game element such as combat, players can experience single player combat (player against the game's artificial intelligence), team-based multiplayer combat (several players teamed against the game's artificial intelligence), or competition-based multiplayer combat (players competing against other players). Choosing among the potential styles of play is also an important game design decision. This is why we include game playing styles as the second dimension of our framework.

\section{A TAXONOMY OF GAME ELEMENTS AND GAME PLAYING STYLES}

After defining a conceptual framework of player preferences, we created a taxonomy of game elements and game playing styles based on an empirical study, which we describe in this section.

\section{Methodology}

We conducted this study to understand participants' preferences for diverse game elements and game playing styles.

\section{Survey Design}

To create the lists of game elements and game playing styles for our survey, we consulted several titles from the game design literature $[1,10,15,31]$. However, we did not find lists that we could use because the literature usually focuses on high level motivations or aesthetics or on low level game mechanics, but we needed a list of elements described in an intermediate granularity level. Therefore, we conducted a brainstorming between three player experience researchers to create lists of game elements and game playing styles. The brainstorming followed an informal process to generate intermediate level elements from the high or low level elements found in the literature and the researchers' experience. Initially, one researcher consulted the mentioned literature with the goal of listing intermediate level elements that could represent the high level elements found in the literature with more details, as well as intermediate level elements that could summarize the low level game mechanics described in the literature. Next, the three researchers reviewed and discussed this initial list of game elements and game playing styles and brainstormed additional elements that they felt were missing from the list, based on their experience with games. The final lists contains 66 game elements and 20 game playing styles. We have provided the complete lists in the appendix, which includes information about each item's descriptions as presented in the survey.

\section{Survey Instrument}

The survey was deployed as an online instrument between February and March 2017 using the LimeSurvey software version 2.50+ (LimeSurvey, 2016). All questions were in English and were grouped as follows:

Preferred game elements: We asked how much participants enjoyed the 66 different game elements in a 5-point Likert scale. The elements were presented in randomized order.

Preferred game playing styles: We asked how much participants enjoyed 20 different game playing styles in a 5-point Likert scale. The styles were presented in randomized order.

Other: Additional questions, unrelated to the current study.

Demographic information: Participant's country, language, age, gender, education, and gaming habits and preferences.

The survey could be completed anonymously and allowed participants to skip any question. Prior to the decision to participate, participants were presented with an online informed consent form. In addition, all the long question groups (such as game elements and game playing styles) had attention check questions (e.g., "Please select ' 3 ' in this item to show us that you are carefully reading all questions.") to verify if participants were reading all the items with attention.

\section{Participants}

We recruited participants by email (in both academic and nonacademic environments), social networks (Facebook, Twitter, and Reddit), and online gaming forums. Participants were required to be at least 15 years old. Study volunteers were 
not offered a direct remuneration, but instead were offered an opportunity to enter a draw to win one of two $\$ 50$ prizes.

In total, 196 participants completed the survey. However, we discarded eight participants who did not complete all question groups or failed to select the correct answer in at least one of the attention check items. Therefore, the final dataset contained 188 responses (124 men, 53 women, 4 transgender, 3 non-binary, and 4 did not inform their gender). Participants' ages ranged from 15 to $71(M=26.7, S D=9.7)$ and were skewed towards younger participants (with $74 \%$ of participants being 30 or less), possibly because the topic of the survey was more appealing to a younger audience.

Participants were distributed geographically as follows: $60.6 \%$ from North America, 25.5\% from Europe, 5.3\% from South America, $4.8 \%$ from Oceania, $2.7 \%$ from Asia, and $1.1 \%$ from Africa. However, $98.9 \%$ of participants reported having a very good or native understanding of English. Therefore, we operate under the assumption that lack of English proficiency was not a detriment to our study.

\section{Analytic Procedure}

In the following subsections, we will present the results of the data analysis procedures that led us to devise a taxonomy of game elements and game playing styles. First, we conducted an exploratory factor analysis to construct the taxonomy. Following the successful factor analysis, we conducted a correlation analysis to determine how the independent variables (gender and age) influence the groups' scores. All analyses were conducted in SPSS 23 (IBM, 2015). We present the details of each of these analyses.

We employed a principal component analysis (PCA) to cluster the surveyed game elements and game playing styles into groups. PCA is a standard method for creating groupings in data based on the covariance and correlation of items. This allowed us to establish a taxonomy and analyze players preferences with a more manageable number of categories. Since our investigation was exploratory and we included game elements and playing styles in our survey through brainstorming, we had no prior theory to justify their inclusion or exclusion from the taxonomy. Therefore, the first step was to evaluate if all the included elements and playing styles could be successfully grouped into clusters. A PCA requires variables to be at least partially correlated between themselves to be able to reduce the number of components. Thus, we first analyzed the correlation matrix between all variables and removed variables with only three or less relevant correlations. We considered correlations with $r>=.3$ as relevant, as suggested by Field [14] (p. 648). Moreover, we also performed an initial PCA and noted the variables that appeared isolated in one of the components (variables that were the only item with a high loading in one of the components and did not have a high loading in any other component) and also removed them. We performed this procedure iteratively, until we found no more variables to remove.

Moreover, we used parallel analysis and Velicer's minimum average partial (MAP) test to determine the number of components to retain in the final analyses because these procedures are validated and, hence, more adequate than a simple eigenvalues inspection [25]. Furthermore, we employed an Oblimin rotation because we expected that the components could partially overlap. Finally, we considered factor loadings greater than .36 as significant, following Field's [14] (p. 644) recommendation for a sample size of 200 and an $\alpha=.01$.

After completing the PCA, we analyzed the composition of each component to interpret and label them. To achieve this, four researchers independently interpreted the structure matrices. Next, they compared their interpretations by discussing similarities and divergences, and came to an agreement on a representative label for each component.

Finally, to understand which factors influence user preferences for each one of the groups in the taxonomy, we analyzed how the participant's gender and age influenced their scores for each group. We included only the genders 'male' and 'female' in the analysis because we did not have enough data points in the remaining categories to perform a statistical analysis. For these correlation analyses, we computed participants' standardized scores for each component as part of the PCA using the regression method. Standardizing the linear regression model generates scores for each component with a mean of zero and a standard deviation of 1.0.

\section{Game Elements}

On completion of the analytic procedure to identify the variables that were useful for the PCA, we removed six game elements from the analysis (9.1\%): fixed rewards, performancebased rewards, random rewards, open progression, horror, and stealth play. This means that these variables could not be clustered with any of the other variables in the dataset. For the final dataset with the remaining 60 variables, the KaiserMeyer-Olkin measure verified the sample adequacy for the analysis, $K M O=.70$ (a good sample size, according to Field [14]). Moreover, Bartlett's Test of Sphericity was significant $\left(\chi^{2}{ }_{1770}=5085.1, p<.001\right)$, indicating that the correlations between items were sufficiently large for PCA. The final results of the PCA revealed nine components. Table 1 presents the final structure matrix.

All components showed adequate reliability with $\alpha>=.665$, except for component 9, which showed a lower reliability of $\alpha=.555$ (see Table 1), but still acceptable. Furthermore, component 9 showed one significant variable (drawing or painting) with a positive sign, whereas all other significant variables were negative. This means that participants who said they enjoy all the other significant elements in this component are very unlikely to also enjoy drawing or painting. Thus, this element was not considered as part of this component.

\section{Component Interpretation}

We labelled the nine components as follows:

1. Strategic resource management: represents several dynamics of resource management, construction, or strategic gameplay.

2. Puzzle: includes diverse types of puzzles.

3. Artistic movement: includes several types or artistic expression (such as music or painting) or body movement. 


\begin{tabular}{|c|c|c|c|c|c|c|c|c|c|}
\hline \multirow[b]{2}{*}{ Game Design Elements } & \multicolumn{9}{|c|}{ Components } \\
\hline & 1 & 2 & 3 & 4 & 5 & 6 & 7 & 8 & 9 \\
\hline $4 \mathrm{X}$ (explore, expand, exploit, and exterminate) & .775 & & & & & & & & \\
\hline Impersonal power & .749 & & & & & & & & \\
\hline Management simulation & .746 & & & & & & & & \\
\hline Unit building & .728 & & & & & & & & \\
\hline Production management & .704 & & & & & & & & \\
\hline Territorial conquest & .663 & & & & & & & .409 & \\
\hline Turn-based strategy & .635 & & & & & & & & \\
\hline Wargame & 607 & & & & & & & .598 & \\
\hline Construction & .569 & & & & & .375 & .396 & & \\
\hline Turn-based combat & .521 & & & & & & & & -.393 \\
\hline Real-time strategy & .483 & & & & & & & .379 & -.379 \\
\hline Sales management & .479 & & & -.429 & & & & & \\
\hline Board game & .445 & & & -.383 & & & & & \\
\hline Spatial puzzle & & .818 & & & & & & & \\
\hline Logic puzzle & & .771 & & & & & & & \\
\hline Strategic escape & & .756 & & & & & & & \\
\hline Maze & & .668 & & & & & & & \\
\hline Tile-matching puzzle & & .531 & .460 & & & & & & \\
\hline Dancing & & & .811 & & & & & & \\
\hline Singing & & & .723 & & & & & & \\
\hline Music & & & .706 & & & & & & \\
\hline Exergaming & & & 695 & & & & & & \\
\hline Drawing or painting & & & .566 & & & & & & .392 \\
\hline Platforms & & .421 & .473 & & & & & .368 & \\
\hline Sports & & & & -.684 & & & & .383 & \\
\hline Sport tournaments & & & & -.682 & & & & .363 & \\
\hline Gambling & & & & -.637 & & & & & \\
\hline Cards & & & & -.613 & & & & & \\
\hline Trivia & & & & -.488 & & & & & \\
\hline Trading & & & & -.482 & & .478 & & & \\
\hline Action-adventure & & & & & .665 & & & .370 & \\
\hline Adventure & & .469 & & & .609 & & & & \\
\hline Fantasy & & & & & .579 & & & & \\
\hline Role-playing & & & & & .570 & & & & \\
\hline Science-fiction & & & & & .540 & & & & \\
\hline Avatar & & & & & .432 & .384 & & & \\
\hline Completion & & & & & .418 & .379 & & & \\
\hline Exploration & & & & & .413 & & & & \\
\hline Collecting & & & & & & .704 & & & \\
\hline Crafting & & & & & & .683 & & & \\
\hline Currency & & & & & & .664 & & & \\
\hline Resource harvesting & .392 & & & & & .571 & & & \\
\hline Equipment management & & & & & & .513 & & & \\
\hline Reality-bound & & & & & & & .748 & & \\
\hline Open world & & & & & & & .636 & & \\
\hline Interactive story & & & & & .450 & & .588 & & \\
\hline Life simulation & & & & & & & .511 & & \\
\hline Shooting & & & & & & & & .727 & \\
\hline Detonation & & & & & & & & .725 & \\
\hline Real-time combat & & & & & & & & .721 & \\
\hline Combat & & & & & & & & .721 & \\
\hline Fighting & & & & & & & & .695 & \\
\hline Racing & & & & -.415 & & & & .632 & \\
\hline Rushed escape & & & & & & & & .627 & \\
\hline Vehicle simulation & & & & & & & & 610 & \\
\hline Parkour & & & .382 & & & & & .548 & \\
\hline Obstacle running & & & .400 & & & & & .478 & \\
\hline Pre-determined progression & & & .372 & & & & & & -.543 \\
\hline Skill trees & & & & & & .400 & & & -.521 \\
\hline Character power & & & & & .404 & .439 & & & -.510 \\
\hline Internal reliability $(\alpha)$ & .878 & .804 & .812 & .761 & .770 & .763 & .665 & .882 & .555 \\
\hline Eigenvalues & 8.651 & 5.572 & 4.604 & 4.105 & 2.735 & 2.546 & 2.097 & 1.980 & 1.665 \\
\hline$\%$ of variance & 14.418 & 9.286 & 7.673 & 6.841 & 4.558 & 4.243 & 3.496 & 3.300 & 2.775 \\
\hline
\end{tabular}

Notes. Extraction method: Principal component analysis. Rotation method: Oblimin with Kaiser normalization.

Principal components were labelled: 1-strategic resource management, 2-puzzle, 3-artistic movement, 4-sports and cards, 5-role-playing, 6 -virtual goods, 7-simulation, 8-action, 9-progression. For improved visualization, the factor loadings $<.36$ (absolute values) are suppressed.

Table 1. Exploratory factor analysis (structure matrix) of the game design elements. 
4. Sports and cards: includes dynamics related with sports, cards, and gambling.

5. Role-playing: represents several dynamics which support role-playing, such as fantasy or science fiction, avatars, and exploration.

6. Virtual goods: includes dynamics of acquisition, collection, and use of virtual goods or resources.

7. Simulation: represents the means by which players can simulate scenarios inspired by real life.

8. Action: includes diverse dynamics related with action, excitement, and fast-paced play.

9. Progression: represents means of progressing towards accumulating power or learning.

Table 2 presents the descriptive statistics for the nine groups of game elements. Overall, role-playing and simulation are the groups that scored higher in player preferences, whereas sport and cards and artistic movement are the groups that scored lower. There is a 1.37-point difference out of $5.0(27 \%)$ in the difference between the highest and the lowest scoring groups.

Table 2 also presents the bivariate correlations of the nine groups between themselves. There is only one significant moderate correlation between puzzle and artistic movement.

\section{Factors that Influence Player Preferences}

Table 3 shows that women scored significantly higher than men for puzzle, artistic movement, and virtual goods, whereas men scored significantly higher for strategic resource management, sports and cards, and action. Regarding age, only the preferences for role-playing and progression seem to significantly decrease with age.

\section{Game Playing Styles}

On completion of the analytic procedure to identify the variables that were useful for the PCA, only one variable (5\%) was removed from the analysis: Casual gaming. This means that the preference for casual gaming stands alone, not grouped with any other styles of playing. For the final dataset with the remaining 19 variables, $K M O=.82$ (meaning that the sample size was great for the analysis, according to Field [14]). Moreover, Bartlett's test of sphericity was significant $\left(\chi^{2}{ }_{171}=1047.0, p<.001\right)$, indicating that the correlations between items were sufficiently large for PCA. The final results of the PCA revealed four components. Table 4 presents the final structure matrix. All components showed a good reliability with $\alpha>=.698$, except for component 3 , which showed a lower $\alpha=.582$, but still acceptable.

\section{Component Interpretation}

We labelled the four components as follows:

1. Multiplayer: includes several types of multiplayer interactions (collaborative or competitive).

2. Abstract interaction: represents individual playing styles in which the player feels less directly immersed in the game, by playing for example from an isometric or a top-down view.

3. Solo play: represents individual playing styles in which the player might be more directly immersed in the game, by playing from a third-person view and freely moving around the game world.

4. Competitive community: represents several ways in which players can interact with others in a community, such as streaming, competing in e-sports, or co-located play.

\begin{tabular}{|c|c|c|c|c|c|c|c|c|c|c|c|}
\hline \multirow[b]{2}{*}{ Components } & & & \multicolumn{9}{|c|}{ Correlations $(r)$} \\
\hline & Mean & SD & 1 & 2 & 3 & 4 & 5 & 6 & 7 & 8 & 9 \\
\hline $\begin{array}{l}\text { 1- Strategic resource management } \\
\text { 2- Puzzle }\end{array}$ & 3.60 & 0.68 & -033 & - & & & & & & & \\
\hline $\begin{array}{l}\text { - Puzzie } \\
\text { 3- Artistic movement }\end{array}$ & $\begin{array}{l}3.65 \\
3.02\end{array}$ & $\begin{array}{l}0.13 \\
0.70\end{array}$ & $\begin{array}{l}-.033 \\
-.030\end{array}$ & $254^{\text {*** }}$ & - & & & & & & \\
\hline 4- Sports and Cards & 2.94 & 0.64 & .155 & .032 & .131 & - & & & & & \\
\hline 5- Role-playing & 4.31 & 0.47 & -.038 & .114 & .008 & -.063 & - & & & & \\
\hline 6- Virtual goods & 3.87 & 0.49 & .119 & .080 & .023 & .016 & .126 & - & & & \\
\hline 7- Simulation & 4.00 & 0.60 & .116 & .063 & .041 & .001 & .032 & .103 & - & & \\
\hline 8- Action & 3.42 & 0.64 & .090 & .046 & .015 & .087 & .082 & .054 & .075 & - & \\
\hline 9- Progression & 3.88 & 0.56 & .131 & -.007 & -.011 & .109 & .104 & .026 & -.067 & .095 & - \\
\hline
\end{tabular}

Table 2. Descriptive statistics for the groups of game elements and bivariate correlations of the groups between themselves.

\begin{tabular}{|c|c|c|c|c|c|c|c|c|c|c|}
\hline \multirow[b]{2}{*}{ Components } & \multicolumn{2}{|c|}{ Female } & \multicolumn{2}{|c|}{ Male } & \multicolumn{3}{|c|}{ t-test } & \multicolumn{2}{|c|}{ Mean Difference } & \multirow[b]{2}{*}{ Age $(r)$} \\
\hline & M & SD & M & SD & $\mathbf{t}$ & df & p & M & SD & \\
\hline 1- Strategic resource management & -0.280 & 1.025 & 0.123 & 0.969 & -2.290 & 143 & .024 & -0.403 & 0.176 & .034 \\
\hline 2- Puzzle & 0.353 & 0.910 & -0.147 & 0.982 & 2.921 & 143 & .004 & 0.500 & 0.171 & 017 \\
\hline 3- Artistic movement & 0.412 & 1.052 & -0.178 & 0.921 & 3.429 & 143 & .001 & 0.590 & 0.172 & -.018 \\
\hline 4- Sports and Cards & -0.276 & 0.892 & 0.154 & 1.028 & -2.441 & 143 & .016 & -0.430 & 0.176 & .005 \\
\hline 5- Role-playing & 0.243 & 1.047 & -0.088 & 0.985 & 1.845 & 143 & .067 & 0.331 & 0.179 & $-.216 * *$ \\
\hline 6- Virtual goods & 0.222 & 0.953 & -0.148 & 0.952 & 2.176 & 143 & .031 & 0.370 & 0.170 & -.141 \\
\hline 7- Simulation & 0.186 & 0.871 & -0.076 & 1.057 & 1.463 & 143 & .146 & 0.262 & 0.148 & -.021 \\
\hline 8- Action & -0.602 & 1.019 & 0.374 & 0.729 & -6.580 & 143 & .000 & -0.976 & 0.148 & $-.3966^{* *}$ \\
\hline 9- Progression & 0.070 & 1.102 & 0.012 & 0.958 & 0.328 & 143 & .744 & 0.059 & 0.179 & .008 \\
\hline
\end{tabular}

Table 3. Independent samples t-test between the groups of game elements and gender and correlations with age. 


\begin{tabular}{|c|c|c|c|c|}
\hline \multirow[b]{2}{*}{ Game Playing Styles } & \multicolumn{4}{|c|}{ Components } \\
\hline & 1 & 2 & 3 & 4 \\
\hline Chatting & .783 & & & .387 \\
\hline Raids & .779 & & & \\
\hline Teams & .759 & & & .439 \\
\hline Voice-over & .728 & & & .471 \\
\hline $\begin{array}{l}\text { Massive multiplayer online } \\
\text { (MMO) }\end{array}$ & .702 & & & \\
\hline $\begin{array}{l}\text { Multiplayer online battle } \\
\text { arena (MOBA) }\end{array}$ & .629 & & & .423 \\
\hline Isometric view & & .789 & & \\
\hline Top-down view & & .761 & & \\
\hline Controlled movement & & .741 & & \\
\hline Third-person view & & .539 & .414 & \\
\hline Player vs. environment & & & .730 & \\
\hline Free movement & & & .706 & \\
\hline Soloing & & & .693 & \\
\hline Streaming & .590 & & & .707 \\
\hline eSports & .457 & & & .667 \\
\hline First-person view & & & & .651 \\
\hline Watching & & & & 647 \\
\hline Co-location or couch co-op & & & & .621 \\
\hline Player vs player & .484 & & & .599 \\
\hline Internal reliability $(\alpha)$ & .872 & .698 & .582 & .857 \\
\hline Eigenvalues & 5.365 & 2.196 & 1.744 & 1.332 \\
\hline$\%$ of variance & 28.234 & 11.558 & 9.179 & 7.011 \\
\hline
\end{tabular}

Notes.

Extraction method: Principal component analysis. Rotation method: Oblimin with Kaiser normalization. Principal components were labelled: 1-multiplayer, 2-abstract interaction, 3-solo play, 4-competitive community. For improved visualization, the loadings $<.36$ (absolute values) are suppressed.

Table 4. Factor analysis (structure matrix) of the game playing styles.

Table 5 presents the descriptive statistics for the groups. We also included casual play because the PCA showed that it cannot be grouped with other styles, but rather it represents a distinct player preference on its own. Overall, solo play is the highest scoring group, whereas multiplayer and competitive community are the lowest scoring groups. The difference between the highest and the lowest scoring groups is 1.06

\begin{tabular}{l|cc|ccccc} 
& \multicolumn{8}{|c|}{ Correlations $(r)$} & \\
Components & $\mathbf{M}$ & SD & $\mathbf{1}$ & $\mathbf{2}$ & $\mathbf{3}$ & $\mathbf{4}$ & $\mathbf{5}$ \\
\hline 1- Multiplayer & 3.11 & 0.86 & - & & & & \\
2- Abstract & 3.46 & 0.67 & .123 & - & & & \\
interaction & & & & & & & \\
3- Solo play & 4.17 & 0.52 & .066 & .080 & - & & \\
$\begin{array}{l}\text { 4- Competitive } \\
\text { community }\end{array}$ & 3.11 & 0.86 & $.359^{* *}$ & .103 & .047 & - & \\
5- Casual play & 3.28 & 1.12 & .071 & .135 & -.072 & -.076 & - \\
\hline$* * p<.01$. & & & & & & &
\end{tabular}

Table 5. Descriptive statistics for the groups of game playing styles and bivariate correlations of the groups between themselves.

points out of $5.0(21 \%)$. Finally, Table 5 also presents the bivariate correlations of the five game playing styles between themselves. The only significant correlation occurs between multiplayer and competitive community, demonstrating that these two playing styles are strongly correlated.

\section{Factors that Influence Player Preferences}

Table 6 presents the results of the t-test of the groups' scores between genders, as well as the correlations with age. Women scored significantly higher than men only for casual play, whereas men scored significantly higher for multiplayer and competitive community. The difference was not significant for abstract interaction and solo play. Regarding age, the preferences for solo play and competitive community seem to significantly decrease with age, whereas the preference for casual play seems to significantly increase with age.

\section{Game Elements vs Game Playing Styles}

There are several significant correlations between the groups of game elements and game playing styles (see Table 7). Figure 1 summarizes the final player preferences model emerged from the data, which consists on the combination of the two taxonomies (game elements and game playing styles) and the correlations between them found in the data.

\begin{tabular}{l|cc|cc|ccc|cc|c} 
& \multicolumn{2}{|c}{ Female } & \multicolumn{2}{c|}{ Male } & \multicolumn{3}{c|}{ t-test } & Mean Difference & Age $(r)$ \\
\hline Components & M & SD & M & SD & t & df & p & M & SD & Age \\
\hline 1- Multiplayer & -0.261 & 1.1232 & 0.181 & 0.913 & -2.628 & 154 & .009 & -0.442 & 0.168 & -.125 \\
2- Abstract interaction & -0.169 & 1.022 & 0.101 & 0.988 & -1.584 & 154 & .115 & -0.270 & 0.170 & .111 \\
3- Solo play & -0.043 & 1.167 & 0.008 & 0.911 & -0.301 & 154 & .764 & -0.051 & 0.171 & $-.156^{*}$ \\
4- Competitive community & -0.517 & 0.874 & 0.254 & 0.967 & -4.823 & 154 & .000 & -0.772 & 0.160 & $-.237^{* *}$ \\
5- Casual play & 3.623 & 1.096 & 3.138 & 1.096 & 2.689 & 174 & .008 & 0.484 & 0.180 & $.157^{*}$ \\
\hline
\end{tabular}

$* p<.05 . \quad * * p<.01$.

Table 6. Independent samples t-test between the groups of game playing styles and gender and correlations with age.

\begin{tabular}{|c|c|c|c|c|c|}
\hline Components & 1- Multiplayer & 2- Abstract interaction & 3- Solo play & 4- Competitive community & 5- Casual play \\
\hline 3- Artistic movement & -.002 & $.184^{*}$ & $-.318^{* *}$ & .017 & $.232^{* *}$ \\
\hline 5- Role-playing & .019 & $.166^{*}$ & $.367^{* *}$ & -.065 & .137 \\
\hline 6- Virtual goods & $.182 *$ & .039 & $.257^{* *}$ & .143 & .095 \\
\hline 7- Simulation & .093 & $-.177^{*}$ & .141 & -.137 & .025 \\
\hline
\end{tabular}

$* p<.05 \quad * * p<.01$.

Table 7. Bivariate correlations between the groups of game design elements and the groups of game playing styles. 


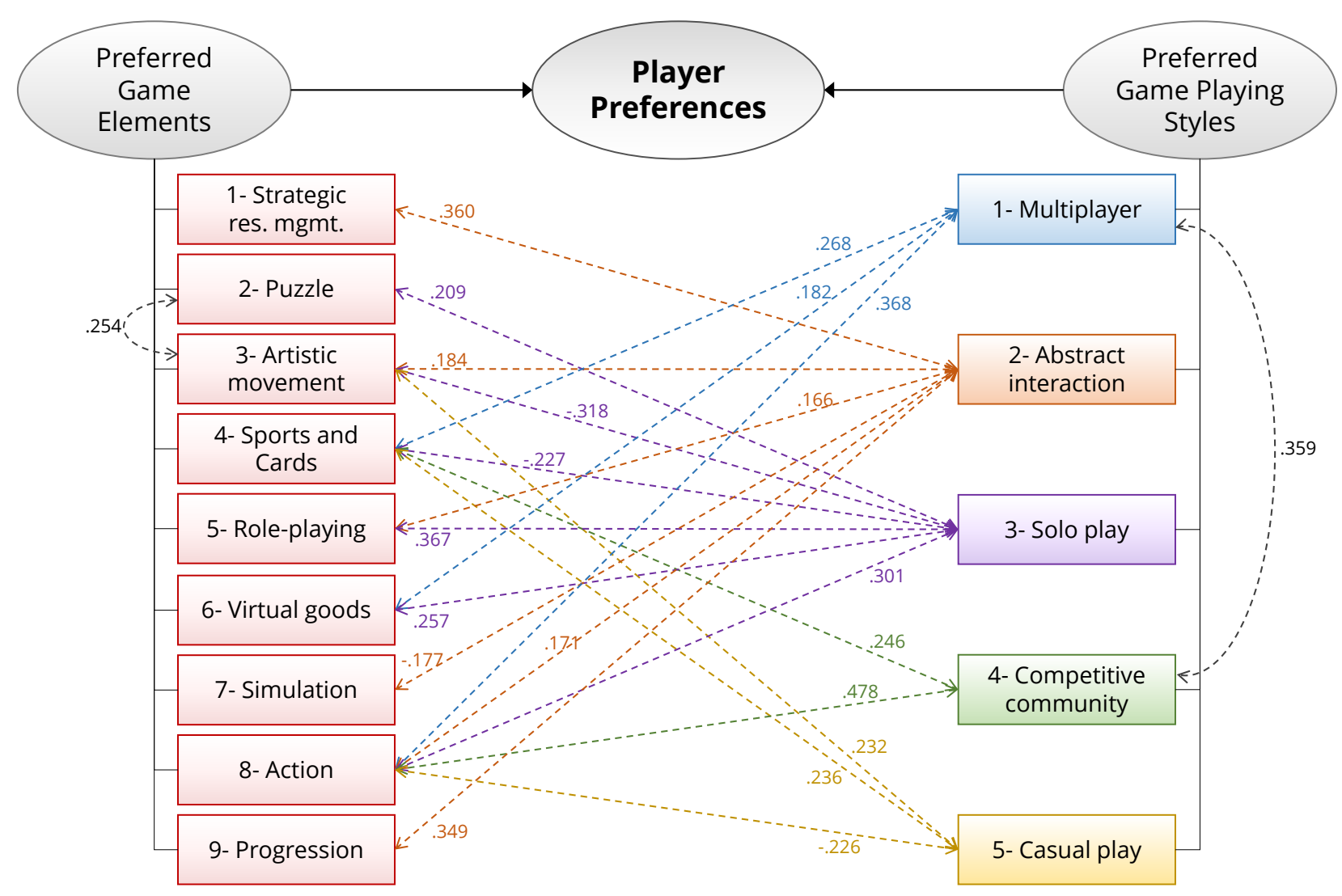

Figure 1. The final player preferences model that emerged from the data. The dashed lines represent the significant correlations $(r)$.

\section{DISCUSSION}

We contribute to the GUR community by proposing a novel conceptual framework of player preferences linking game elements and game playing styles. Without reservation, we demonstrate that there are discernible patterns of player preferences, which can be used by designers and researchers to inform game design and evaluation. For instance, one of the patterns identified by our study is the relationship between game elements and game playing styles, such as 'action, multiplayer, and competitive community' or 'solo play and roleplaying'. Furthermore, our results show that players' gender and age significantly influence their preferred game elements and playing styles.

Designers can benefit from our findings by using them to inform the creation of tailored games, which might be more successful in engaging the targeted players. For example, a game studio might screen its target audience and find that players enjoy some of the strategic resource management elements, such as unit building and production management. Thus, it is likely that these players will also enjoy all other elements from the same group. Therefore, designers can focus on integrating additional elements from this group, such as territorial conquest and turn-based strategy, to create a game that is strongly appealing to the target players. In addition, designers can assume that these players will likely enjoy the abstract interaction style of play because it is positively correlated with strategic resource management. Similarly, researchers can use the taxonomy to better understand and identify mediating factors of player experience. For example, a researcher studying player experiences in action games might measure participants' preferences for these kind of elements, then verify if the difference in participants' preferences for action elements influences their experiences.

Furthermore, these findings are important for serious games because they are usually meant to engage a broad player audience into achieving an instrumental goal. However, if a player does not enjoy the game, it will be difficult to achieve the goals. By better understanding player preferences, designers can better tailor their serious games to their target audience, or focus on the elements and playing styles that are enjoyed by a larger number of players.

\section{Factors of Influence in Player Preferences}

Regarding the differences observed in participants' overall enjoyment of each group of game elements and playing styles, it is worth noting that role-playing, simulation, and solo play are the highest scoring groups. Therefore, game designers can use this information as a guideline to inform their ideation process because our findings show that games which employ these elements are more likely to appeal to a larger audience.

Considering the factors that influence player preferences, gender appears to be the most significant factor. Women appear to 
enjoy more casual play, as well as puzzles, artistic movement, and virtual goods elements. On the other hand, men appear to enjoy more multiplayer gaming and competitive communities, as well as strategic resource management, sports and cards, and action elements. Contrarily, solo and abstract interaction playing styles, as well as role-playing, simulation, and progression elements are similarly appealing to both women and men. Williams et al. [34] had previously noted that women are more motivated by social reasons, whereas men are more motivated by achievement-related reasons. Our findings present a new perspective on this topic because we have looked at game elements in more detail. Thus, we show that women are more likely to enjoy puzzles, which might be seen as one form of achievement-related motivation. In comparison, our findings demonstrate that men are more likely to enjoy multiplayer gaming and competitive communities. Therefore, we can conclude that men seem to be more attracted to the challenge of playing with or against other players, rather than the social interactions.

On the other hand, age does not explain much of the variability in player preferences, but there are some important considerations. Our findings suggest that games targeting older audiences should lean more towards casual games that are easier to learn and play. On the contrary, games focused on action elements are more likely to appeal to younger audiences.

Finally, it is possible to identify some relationships between game elements and game playing styles: (1) action, sports, and cards strongly favour multiplayer and competitive communities; (2) progression and strategic resource management elements strongly favour the abstract interaction playing style; (3) role-playing, action, and virtual goods favour solo playing; and (4) casual gaming occurs more frequently with artistic movement and sports and cards elements. Therefore, designers can use this information as guidelines for building more entertaining games because these combinations are more likely to appeal to their targeted player audience.

\section{Relationship of our Framework with Related Work}

We attempted to compare our taxonomy with the existing models from the literature: Yee's gamer motivation profile [36], the BrainHex [24], Hamari and Tuunanen's dimensions [17], and Ferro et al.'s categories [13]. However, we concluded that the groups identified in our taxonomy do not seem to be directly associable to the dimensions in these models. This is because our model does not identify player types or traits; it groups game elements and game playing styles according to how likely it is for players to enjoy them. Nonetheless, one question remains: how do the groups of game elements and game playing styles that we uncovered relate to the dimensions of motivations and player archetypes suggested by the extant literature? Further studies are needed to answer this question.

\section{Limitations and Future Work}

We note all measures were self-reported and, thus, subject to participants' level of understanding and their awareness of their own preferences. Moreover, the present study was exploratory, aimed at constructing an initial conceptual framework and taxonomy of player preferences. Therefore, there is room for additional studies with larger samples to verify our findings and confirm the validity of our framework and taxonomy. Furthermore, because there is currently no universal and agreed upon list of game elements, we devised our own list of game elements and game playing styles for this study. Consequently, our results are particular to the characteristics of the list we employed. Regardless, we are confident in the validity of our model as a good representation of player preferences because our list was based on the extant game design literature and encompassed a bigger variety of game elements than what has been seen in studies of player preferences so far. The goal of the presented research was to look at self-reported preferences. However, future studies could investigate the relationship between an individual's self-reported preferences and their actual behaviour.

\section{CONCLUSION}

In conclusion, we contributed a new conceptual framework and created a taxonomy of game elements and game playing styles based on an empirical investigation of player preferences. We also showed that a player's gender and age significantly influences their average preferences: women are more inclined to enjoy casual play, artistic play, puzzles, and virtual goods, whereas men are more inclined to enjoy strategy, sports, cards, action, multiplayer and competitive play. Additionally, younger players are more likely to enjoy action, role-playing, solo play, and competition, whereas older players are more likely to enjoy casual play.

The strength of the presented work stems from the fact that it is the first in the literature that explores player preferences towards the building blocks of games, instead of mapping abstract player motivations that do not translate directly into game design suggestions. Therefore, we contribute to the HCI and GUR communities by providing a practical framework, which would empower designers to create more engaging games, as well as helping researchers better understand player experiences and the effects of games on players. For example, the designers of a MMO game might use our framework with the goal of further expanding their community, by understanding what are the elements and playing styles that their players are more likely to enjoy to make more informed decisions.

Additionally, this research has implications for the design of serious games. Since our framework classifies different player preferences in a way that can be directly mapped into design decisions, it would help serious games designers to create experiences that are appealing to a broad audience or to specific groups of players. Therefore, our work will assist on creating more engaging and effective serious games.

\section{ACKNOWLEDGMENTS}

Gustavo Tondello would like to thank the University of Waterloo and the CNPq, Brazil for his funding. Rina Wehbe would like to thank the University of Waterloo and NSERC for her funding. Rita Orji would like to thank the Canadian Government Banting NSERC for her funding. This research has received funding from NSERC (RGPIN-418622-2012), SSHRC (895-2011-1014, IMMERSe), CFI (35819), Mitacs (IT07255), and SWaGUR. 


\section{REFERENCES}

1. Ernest Adams and Joris Dormans. 2012. Game Mechanics: Advanced Game Design. New Riders, Berkeley, CA.

2. Richard Bartle. 1996. Hearts, Clubs, Diamonds, Spades: Players who suit MUDs. Journal of MUD Research 1, 1 (1996).

3. Richard Bartle. 2005. Virtual Worlds: Why People Play. Massively Multiplayer Game Development 2, 1 (2005).

4. Chris Bateman and Richard Boon. 2006. $21^{\text {st }}$ Century Game Design (Game Development Series). Charles River Media.

5. Chris Bateman, Rebecca Lowenhaupt, and Lennart E Nacke. 2011. Player Typology in Theory and Practice. Proceedings of DiGRA 2011 (2011).

6. Chris Bateman and Lennart E Nacke. 2010. The Neurobiology of Play. In Proceedings of Futureplay 2010. ACM, Vancouver, BC, Canada, 1-8. DOI : http://dx. doi .org/10.1145/1920778.1920780

7. Max V. Birk, Cheralyn Atkins, Jason T. Bowey, and Regan L. Mandryk. 2016. Fostering Intrinsic Motivation through Avatar Identification in Digital Games. In Proceedings of the $34^{\text {th }}$ Annual ACM Conference on Human Factors in Computing Systems. ACM. DOI : http://dx.doi.org/10.1145/2858036.2858062

8. Max V. Birk, Dereck Toker, Regan L. Mandryk, and Cristina Conati. 2015. Modeling Motivation in a Social Network Game Using Player-Centric Traits and Personality Traits. In Proceedings of User Modeling, Adaptation and Personalization. Springer, 18-30. DOI: http://dx.doi.org/10.1007/978-3-319-20267-9_2

9. Blizzard Entertainment. 2004. World of Warcraft. Game [Windows/OS X]. (2004).

10. Brenda Brathwaite and Ian Schreiber. 2009. Challenges for Game Designers. Charles River Media, Boston, MA.

11. Marc Busch, Elke Mattheiss, Rita Orji, Peter Fröhlich, Michael Lankes, and Manfred Tscheligi. 2016. Player Type Models - Towards Empirical Validation. In Proceedings of the 2016 CHI Conference Extended Abstracts on Human Factors in Computing Systems. ACM. DOI : http://dx.doi .org/10.1145/2851581.2892399

12. Edward L Deci and Richard M Ryan. 1985. Intrinsic Motivation and Self-Determination in Human Behavior. Plenum, New York and London.

13. Lauren S. Ferro, Steffen P. Walz, and Stefan Greuter. 2013. Towards personalised, gamified systems: an investigation into game design, personality and player typologies. In Proceedings of the $9^{\text {th }}$ Australasian Conference on Interactive Entertainment: Matters of Life and Death - IE '13. 1-6. DOI : http://dx.doi.org/10.1145/2513002.2513024

14. Andy Field. 2009. Discovering Statistics Using SPSS (3 ${ }^{\text {rd }}$ ed.). Sage Publications, London, UK.
15. Tracy Fullerton. 2008. Game Design Workshop: A playcentric approach to creating innovative games $\left(2^{\text {nd }}\right.$ ed.). Morgan Kaufmann, Burlington, MA.

16. Lewis R Goldberg. 1993. The structure of phenotypic personality traits. American Psychologist 48, 1 (1993), 26. DOI: http://dx.doi.org/10.1037/0003-066X.48.1.26

17. Juho Hamari and Janne Tuunanen. 2014. Player types: A meta-synthesis. Transactions of the Digital Games Research 1, 2 (2014). http://todigra.org/index.php/todigra/article/view/13

18. Robin Hunicke, Marc LeBlanc, and Robert Zubek. 2004. MDA: A Formal Approach to Game Design and Game Research. In Workshop on Challenges in Game AI. 1-4. DOI: http://dx.doi.org/10.1.1.79.4561

19. Shih-Ping Jeng and Ching-I Teng. 2008. Personality and motivations for playing online games. Social Behavior and Personality: an international journal 36, 8 (2008), 1053-1060. DOI :

http://dx.doi.org/10.2224/sbp.2008.36.8.1053

20. Kibeom Lee and Michael C. Ashton. 2004. Psychometric Properties of the HEXACO Personality Inventory. Multivariate Behavioral Research 39, 2 (2004), 329-358. DOI: http://dx.doi.org/10.1207/s15327906mbr3902_8

21. Ian J Livingston, Carl Gutwin, Regan L Mandryk, and Max Birk. 2014. How players value their characters in World of Warcraft. In Proceedings of the $17^{\text {th }}$ ACM conference on Computer Supported Cooperative Work \& Social Computing. ACM, 1333-1343. DOI: http://dx.doi.org/10.1145/2531602.2531661

22. Nicole McMahon, Peta Wyeth, and Daniel Johnson. 2012. Personality and Player Types in Fallout New Vegas. In Proceedings of the $4^{\text {th }}$ International Conference on Fun and Games (FnG '12). ACM, 113-116. DOI: http://dx. doi .org/10.1145/2367616.2367632

23. Isabel Briggs Myers. 1962. The Myers-Briggs Type Indicator. Consulting Psychologists Press, Palo Alto, CA.

24. Lennart E Nacke, Chris Bateman, and Regan L Mandryk. 2014. BrainHex: A Neurobiological Gamer Typology Survey. Entertainment Computing 5, 1 (2014), 55-62. DOI : http://dx.doi .org/10.1016/j. entcom. 2013.06.002

25. Brian P O'Connor. 2000. SPSS and SAS programs for determining the number of components using parallel analysis and Velicer's MAP test. Behavior Research Methods, Instruments, \& Computers 32, 3 (2000), 396-402. DOI : http://dx.doi .org/10.3758/BF03200807

26. Rita Orji, Regan L. Mandryk, Julita Vassileva, and Kathrin M. Gerling. 2013. Tailoring persuasive health games to gamer type. In Proceedings of the SIGCHI Conference on Human Factors in Computing Systems CHI'13. 2467-2476. DOI : http://dx.doi.org/10.1145/2470654.2481341 
27. Rita Orji, Lennart E. Nacke, and Chrysanne DiMarco. 2017. Towards Personality-driven Persuasive Health Games and Gamified Systems. In Proceedings of the SIGCHI Conference on Human Factors in Computing Systems. ACM. DOI :

http://dx.doi.org/10.1145/3025453.3025577

28. Nicole Peever, Daniel Johnson, and John Gardner. 2012. Personality \& Video Game Genre Preferences. In Proceedings of The $8^{\text {th }}$ Australasian Conference on Interactive Entertainment: Playing the System. ACM, 20. DOI : http://dx.doi.org/10.1145/2336727.2336747

29. Andrew K Przybylski, C Scott Rigby, and Richard M Ryan. 2010. A motivational model of video game engagement. Review of general psychology 14, 2 (2010), 154. DOI : http://dx.doi.org/10.1037/a0019440

30. Richard M. Ryan, C. Scott Rigby, and Andrew Przybylski. 2006. The motivational pull of video games: A self-determination theory approach. Motivation and Emotion 30, 4 (2006), 347-363. DOI : http://dx.doi.org/10.1007/s11031-006-9051-8

31. Jesse Schell. 2008. The Art of Game Design: A Book of Lenses. Morgan Kaufmann, Burlington, MA.

32. Gustavo F Tondello, Rina R Wehbe, Zachary O Toups, Lennart E Nacke, and Nicole K Crenshaw. 2015. Understanding Player Attitudes Towards Digital Game Objects. In Proceedings of the 2015 Annual Symposium on Computer-Human Interaction in Play - CHI PLAY' 15. ACM, London, United Kingdom. DOI : http://dx.doi .org/10.1145/2793107.2810292

33. Zachary O. Toups, Nicole K. Crenshaw, Rina R. Wehbe, Gustavo F. Tondello, and Lennart E. Nacke. 2016. "The
Collecting Itself Feels Good": Towards Collection Interfaces for Digital Game Objects. In Proceedings of the 2016 Annual Symposium on Computer-Human Interaction in Play - CHI PLAY'16. ACM, Austin, TX, United States. DOI :

http://dx.doi.org/10.1145/2967934.2968088

34. Dmitri Williams, Mia Consalvo, Scott Caplan, and Nick Yee. 2009. Looking for gender: Gender roles and behaviors among online gamers. Journal of Communication 59, 4 (2009), 700-725. DOI : http://dx.doi.org/10.1111/j.1460-2466.2009.01453.x

35. Nick Yee. 2006. Motivations for Play in Online Games. CyberPsychology \& Behavior 9, 6 (2006), 772-775. DOI: http://dx.doi.org/10.1089/cpb.2006.9.772

36. Nick Yee. 2015. Gamer Motivation Model Overview and Descriptions. Quantic Foundry. (Dec 2015). http://quanticfoundry.com/2015/12/15/handy-reference/

37. Nick Yee. 2016. Gaming Motivations Align with Personality Traits. Quantic Foundry. (Jan 2016). http:// quanticfoundry.com/2016/01/05/personality-correlates/

38. Nick Yee, Nicolas Ducheneaut, and Les Nelson. 2012. Online gaming motivations scale: development and validation. In Proceedings of the 2012 ACM annual conference on Human Factors in Computing Systems CHI' 12. ACM, New York, New York, USA, 2803. DOI : http://dx.doi.org/10.1145/2207676.2208681

39. Virgil Zeigler-Hill and Sean Monica. 2015. The HEXACO model of personality and video game preferences. Entertainment Computing 11 (2015), 21-26. DOI : http://dx. doi .org/10.1016/j.entcom.2015.08.001 